\title{
Intimité de Jean-Victor Pellerin, ou à la recherche du " théâtre du silence "
}

On admire le « Sortez! » de Roxane; mais, à la scène, le mouvement accompagnant la réplique, l'effet diminue parce qu'il se dédouble ; un geste muet aurait une tout autre valeur. Seulement il n'y a de silences qu'au théâtre ; on ne les souffre pas au royaume de Sire le Mot.

G. Baty, $1926: 162$.

Arnaud Rykner constate que « l'idée même que le silence puisse avoir de l'importance au théâtre a longtemps été inconcevable » (2001 : 1525). De fait, la tradition classique française a pesé lourdement sur la dramaturgie en empêchant toute incursion dans l'afflux dialogique. À part les conceptions de Diderot ou la pratique du drame bourgeois, qui faisaient fi des prescriptions rigides du Grand Siècle, ce n'est qu'avec l'avènement des naturalistes et des symbolistes que le silence occupe une place importante dans le théâtre occidental. En France, dans les années 1930, s'affirme sous la houlette de Gaston Baty un mouvement original auquel on a donné l'appellation parlante de "théâtre du silence ». Dénommé aussi bien " théâtre d'évasion " ou « théâtre de l'inconscient ", il regroupait plusieurs écrivains tels que Jean-Jacques Bernard, Denys Amiel, Charles Vildrac, Henri-René Lenormand, Simon Gantillon ou enfin Jean-Victor Pellerin, tous voués à un art illustrant un ensemble de doctrines que le créateur de la Grande Pastorale formulait dans ses écrits programmatiques. Cette expérience, bien accueillie en son temps, n’a pas fait long feu ; néanmoins, l'esthétique " intimiste » a contribué à l'évolution de la dramaturgie pas seulement française. Il est à ce propos intéressant d'évoquer l'une des multiples pièces montées dans

Tomasz Kaczmarek - professeur à l'Institut de Philologie Romane de l'Université de Łódź. Adresse pour correspondance : Institut d’Études Romanes, Université de Łódź, ul. Pomorska 171/173, 91-404 Łódź, Pologne ; e-mail : tomasz.kaczmarek@uni.lodz.pl 
la Baraque, le cas échéant Intimité de Pellerin, pour voir comment le silence n’était pas uniquement utilisé comme un moyen au service du texte, mais aussi comme un élément parfaitement dramatique, déclencheur d'un malaise et manifestant la solitude et le vide ontologique.

Quand Jean-Victor Pellerin (1889-1970) s'apprête à écrire sa première pièce dramatique, il a déjà à son actif quelques textes romanesques et poétiques, dans lesquels il exprime sa volonté d'étudier les remous ombrageux de la vie intérieure de "l'homme moderne ». Dès lors, il se rend compte des carences de la langue, incapable de ramener à la surface tout ce qui reste intimement refoulé. À la même époque, il se fascine pour le théâtre de Gaston Baty, relit Maurice Maeterlinck et devient l'ami proche de Jean-Jacques Bernard. C'est par l'intermédiaire de celui-ci que le jeune écrivain va connaître Baty, à qui il propose son texte : Intimité (1921), accepté sans coupures par le metteur en scène (Lieber, 1987 : 399). Alors, le dramaturge déclare fièrement qu'il veut décrire dans son drame le triomphe " de l'âme errante et passionnée sur l'âme banale et routinière, du rêve sur le réel » (Pellerin, 1922: 70).

Avec cette première œuvre, Pellerin s'inscrit directement dans la poétique qui règne à la Baraque dont les conceptions s'expliquent peut-être le mieux à la lumière $\mathrm{du}$ « tragique quotidien " proposé par Maeterlinck. En effet, l'auteur des Sept princesses rejette le tragique tapageur avec sa violence spectaculaire et sa force anecdotique en mettant l'accent sur un autre tragique qui s'instaure loin des champs de bataille, à l'abri de la maison, quand l'homme est hanté par des forces invisibles. Dans ce contexte, la parole qui voudrait dire toute la vérité semblerait superflue, car elle ne pourrait jamais exprimer ce qui est inexprimable. Néanmoins, "à côté du dialogue indispensable, il y a presque toujours un autre dialogue [...] - comme l'explique le Belge - examinez attentivement et vous verrez que c'est le seul que l'âme écoute profondément " (Maeterlinck, $1986: 107)$. Bernard reprend cette idée : «il y a sous le dialogue entendu comme un dialogue sous-jacent qu'il s'agit de rendre sensible », car, «le théâtre est avant tout l'art de l'inexprimé [...]. Dès que nous avons vraiment quelque chose à dire, nous sommes obligés de nous taire " (Bernard, $1958: 47-48$ ). En s'inspirant ouvertement de cette esthétique, Pellerin scrute "l'indicible » qui se love derrière le discours pléthorique et intempestif tout en valorisant la dimension métaphysique du silence. Ce procédé fait florès, car, comme le note Marcel Raymond «c'est bien souvent au moment où l'homme a le plus à dire qu'il se tait» (1943: 85).

L'action du drame n'est pas compliquée : tout commence dans une salle à manger, où les deux personnages principaux se retrouvent, après le dîner, dans l'intimité, à s'occuper des travaux habituels : la femme tricote, tandis que l'homme lit le journal. Le début de la pièce ne présage rien de novateur et on pourrait légitimement penser à un énième drame bourgeois. De fait, on assiste à un dialogue entre le couple qui s'entretient sur des sujets aussi futiles que banals : Monsieur et Madame ressassent de vieilles histoires insignifiantes qui semblent remplir les heures de lassitude de tous les jours. Cette conversation dépouillée de toute dimension artistique annonce tout de même non seulement la monotonie désespérante qui règne sans partage au quoti- 
dien dans le foyer, mais témoigne aussi de la solitude des personnages qui se parlent sans rien se dire, car leurs répliques ne donnent pas de suite, tous les deux se livrant en réalité à de vrais soliloques. Ce dialogue de sourds arrive au point culminant, après lequel la patience des spectateurs serait mise à l'épreuve. Mais voilà que dans ce monde d'intimité ennuyeuse interviennent des fantômes qui ne perturbent pourtant pas la « quiétude assommante» de la soirée :

Alors, dans l'ombre, en sourdine, une phrase musicale sélève, romance sentimentale et quelque peu sotte du temps où Madame était très jeune : Quand l'amour meurt... et voilà que derrière elle, - bleuâtre, irréelle, - une femme apparaît, qui reste sur place, immobile (Pellerin, $1927: 14$ ).

C'est à partir de ce moment que Madame entame un long dialogue avec le spectre féminin, qui prend aussitôt la forme d'un monologue sans fin. Au cours de cet entretien, la femme formule ses déceptions sur la vie morne qu'elle partage avec son époux indolent. Elle s'apitoie sur son existence qu'elle juge médiocre et dénuée de sens. Ses lamentations, qui peuvent sembler triviales, révèlent la déréliction du protagoniste se manifestant autant par l'impossibilité de communiquer avec son homme. Cette rencontre inopinée donne à la malheureuse la possibilité d'extérioriser les remous de son subconscient :

- Ah! Ma chère Thérèse, si tu savais !...

- Mais oui, tu es la seule, la seule à qui je puisse me confier.

- Toi ! Allons donc, tu ne connais pas ton bonheur! Nulle attache, rien qui te lie, tu as arrangé ta vie selon ton plaisir, et tu es libre, libre !...

La musique se tait.

- Soit ! La vie que tu mènes n'est pas gaie tous les jours.

- Sans personne auprès de toi, je comprends...

- Écoute un peu, tu parles de solitude, mais... et la mienne?

- Oui, oui, j'entends bien : mon mari, mon enfant. C'est ça que tu envies ?... eh bien, ma petite, si je le pouvais, je te les céderais bien volontiers.

- L'intimité !... Ah ! Voilà un mot que Raymond emploie sans cesse. Un mot, pas autre chose, un pauvre mot! (Pellerin, 1927 : 14-15).

Pellerin réussit à exprimer la condition désolante de la femme, même à travers les propos ordinaires de celle-ci. De fait, le dramaturge " modernise " à sa manière la conception du «tragique quotidien » et cherche dans la banalité la manifestation de l'ennui ontologique, désire dramatiser les platitudes de la vie quotidienne, criblée de désirs inavoués. Tout ceci se trouve envoûté par une atmosphère mystérieuse, car, l'écrivain n'hésite pas à chercher même dans une bourgeoisie gâteuse des sentiments nobles. Du point de vue formel, le discours du protagoniste semble sauvegarder les éléments superficiels d'un dialogue : les tirets accentuent à chaque fois la reprise 
d'une idée qui vient à l'esprit de Madame, les points de suspension marquent les pauses qui interrompent le fil conducteur de son soliloque, surtout dans les moments où la parole lui semble insuffisante. Quand l'épouse négligée retarde son discours, on pourrait en déduire tout simplement que ces pauses sont là juste le temps que l'interlocuteur imaginaire puisse répondre à ses questions, mais, en analysant de plus près ses « jérémiades", on se rend compte que la femme discute bel et bien avec ellemême. Alors, conformément aux attributs d'un vrai monologue, Madame s'adresse à sa conscience, évoque les circonstances précaires dans lesquelles elle se trouve, mais son discours est privé (du moins au début) de dimension introspective ou délibérative. C'est par ce biais que l'auteur de 32 Décembre met en exergue l'isolement du protagoniste qui vit à l'écart de la réalité.

Mais le dramaturge ne présente pas uniquement les chagrins de Madame, il donne aussi la parole à Monsieur. Pellerin met en contraste les monologues des deux époux : si la femme incomprise désire une autre vie, le mari paraît grossier et libidineux. Tout en restant vicieux, l'homme ne cache pas son désarroi devant la fuite du temps. Nous le remarquons dans le "dialogue apparent " que Monsieur mène avec un certain Ernest :

- À propos, connais-tu la dernière marotte d'Yvonne ?...

- Figure-toi qu'elle ne rêve plus que musique classique, Comédie-Française, théâtre d’art ! Brr... ! Si je ne protestais pas énergiquement, nous ne sortirions jamais que pour entendre des chefs-d'œuvre. Ah ! Les chefs-d'œuvre, mon vieux, ce qu'il peut y en avoir d'embêtants !

- D'accord. Ce n'est là qu'un détail... Mais, tu sais, rien de tel que les détails pour abîmer un ensemble.

- Ah! Je ne dis pas non! Ton système a du bon!

Ernest commence de s'effacer.

- Certes, le temps passe, on vieillit...

- Ma foi, un peu de jeunesse ne me ferait pas de mal... un peu de jeunesse, de gaîté... (Pellerin, 1927 : 15-16).

Quand nous écoutons ces deux « épaves humaines » qui ruminent sur leur sort peu enviable, il serait permis de constater que leurs «monologues intérieurs » consistent en un discours qu'ils tiennent avec leurs instances psychiques respectives. C'est-àdire que Madame, tout en conversant avec Thérèse, ne parle pas avec sa meilleure amie, mais avec le pâle reflet de l'époque où elle était jeune. Idem pour Monsieur qui s'adresse à Ernest, un jeune homme gaillard symbolisant la force virile que le mari inassouvi a depuis longtemps perdue.

Les personnages semblent ne pas tolérer le silence, c'est pour cette raison qu'ils se livrent à des conversations chimériques censées apaiser leur angoisse. Mais, celle-ci ne disparaît point, étant donné que la langue s'avère insuffisante, puisqu'elle n'a plus de sens. Et sur ce point, il serait tentant de voir dans le drame du Français l'annonce 
de la mystique apocalyptique au théâtre, celle que Beckett exprimera des dizaines d'années plus tard dans son œuvre. Cependant, si les gueux beckettiens capitulent devant le néant, les névrosés de Pellerin se cramponnent avec obsession aux mots, seuls capables de remplir le pressentiment de vacuité. Ils parlent, donc ils sont. Puisque «la parole naît du silence comme le geste de l'immobilité, comme le visible de l'invisible » (Rousseaux, 2003), l'écrivain, paradoxalement, essaie d'exprimer ce qui ne peut pas ou ne veut pas être dit. C'est de cette manière que l'auteur de Maya cherche l'expression des sentiments de ses pauvres hères sous la surface du langage, il inspecte tout ce qui se cache derrière la parole prononcée. On est encore loin de la sous-conversation de Nathalie Sarraute, mais en voulant décrire, ou plutôt, évoquer les mouvements escamotés qui bouleversent la vie des personnages, le dramaturge devine l'existence des tropismes (Sarraute, 1956 : 8). Quoi qu'il en soit, Pellerin, à l'instar de Bernard, tente de dévoiler le silence dissimulé derrière les paroles qui véhiculent le stéréotype, soutenu par la rigidité de la langue. Dans cette perspective, peut-être plus que de silence, il faudrait parler de la recherche de l'inexprimé, comme le signale explicitement l'auteur de Martine :

Je pense pourtant que vous avez compris que le silence, beaucoup plus généralement que les temps entre les répliques, beaucoup plus largement, c'est tout ce que les personnages ne veulent ou ne peuvent dire, c'est toute la série des pensées ou des désirs qui échappent aux mots, qui ne peuvent s'échanger que par allusion indirecte, voire par le regard ou l'attitude, c'est toute la gamme des sentiments inexprimés, inavoués ou inconscients. Et c'est pourquoi, rejetant l'expression de théâtre du silence, j'ai toujours préféré celle de théâtre de l'inexprimé, que j’avais d'ailleurs employé dès la première fois (Bernard, 1931 : 27).

Mais les protagonistes ne s'entretiennent pas seulement avec leurs « doubles » respectifs, d'autres apparences se manifesteront, comme si elles reflétaient leurs envies refoulées. De fait, au cours de cette soirée d'une « quiétude un peu flasque " (Pellerin, 1927 : 5), les deux bourgeois peuplent leurs mondes de spectres qui prêtent exclusivement corps à leurs pensées ou carrément à leurs désirs profonds. Quant à leurs fantasmes intimes, la langue s'avère défaillante et sera bientôt remplacée par des scènes mimiques qui jouent un rôle dramatique important au même titre que les silences ou la musique. À un moment donné, quand Madame parle avec Thérèse, du côté de Monsieur apparait la Bonne. Celle-ci est une gamine charmante et coquette qui, dès son entrée sur scène, lève lascivement sa jupe pour rajuster sa jarretière. Comme l'homme ne peut rester indifférent aux appas de la jeune femme, il se jette maladroitement sur elle, mais elle se sauve telle une chimère d'un autre monde et se dilue dans l'air. Dès sa disparition, du côté de Madame se fait voir aussitôt un boxeur aux muscles bien dessinés qui attire sexuellement la femme à tel point qu'elle rêve de trouver le repos dans ses bras. Vers la fin de la pièce, Pellerin contraste cet espace imaginaire avec l'espace réel : les deux mondes vont se rejoindre sur un ton quelque peu plaisant. Or, une fois les spectres disparus, le mari impatient et surtout bouleversé par les 
charmes de la domestique, invite sa femme à venir se coucher. Celle-ci suit machinalement l'appel de son homme, mais elle entraîne aussi le sportif vers le lit conjugal, elle "l'entraîne rapidement - précisent les didascalies - l'entraîne vers la chambre à coucher - où les autres sont déjà, qui attendent »(Pellerin, 1927 : 36).

Le dramaturge agrémente aussi son drame de quelques scènes comiques, qui frôlent parfois l'absurdité. Ce procédé semble viser à ôter aux monologues incessants leur dimension tragique, mais le comique, au contraire, renforce encore plus la condition précaire des protagonistes enfermés dans une intimité étouffante. Certains critiques ne se trompent pas en observant que le spectacle, réalisé par Baty même, est « beaucoup moins comique qu'il ne paraît d'abord " (Jenkenne, $1923: 115)$. Cette pièce anticipe, toutes proportions gardées, certaines techniques du " Théâtre de l'Absurde " : « la banalité du dialogue, assortie à la matérialisation des obsessions du subconscient, n'est pas sans rappeler La Cantatrice chauve qu'Eugène Ionesco écrira trente ans plus tard " (Vaïs, 1978 : 33). En effet, dès les premières répliques, la pièce regorge de bizarreries de tout acabit, comme si l'auteur voulait de prime abord souligner l'incommunicabilité entre les personnages. Et sur ce plan, il est tout à fait légitime de voir dans cette œuvre l'anticipation de certains procédés prônés par les « absurdistes » des années 50 : phrases décousues, paroles dénuées de substance sémantique, etc. Parfois, les sujets abordés par le couple peuvent étonner ou prêter à rire. À ce moment là, Monsieur parle des Allemands qui auraient réussi à inventer un procédé chimique pour désinfecter et désodoriser les excréments pour en tirer une sorte de farine alimentaire (Pellerin, 1927 : 16). Un Inconnu, qui s'entretient avec Madame, semble comprendre les désillusions de la femme, mais voilà qu'il décide à l'improviste d'aller en Afrique étudier l'âme nègre (pendant une dizaine d'années) en laissant la pauvre " amie " interloquée et déçue (Pellerin, 1927 : 23). Ailleurs, Monsieur, visiblement neurasthénique, pense souffrir de névralgies et s'inquiète sérieusement de sa santé. Madame affirme que les maux du mari sont dus à ses dents défectueuses et, sur ces entrefaites, arrive un dentiste qui examine à la diable la mâchoire du malade présumé (Pellerin, $1927: 23$ ).

Le dramaturge, en mettant en contraste l'espace imaginaire et l'espace réel, mélange aussi avec maîtrise le tragique avec le comique. Le portrait de ses personnages témoigne de son adhésion à la dramaturgie intimiste basée sur l'inexprimé. Ces pauvres bourgeois empêchés de s'exprimer rappellent l'homme tragique dont parlait Maeterlinck. Pellerin choisit des gens peu distingués qui paraissent parfois vulgaires et les place dans les circonstances accablantes de l'ennui. Une vraie fatalité pèse sur eux, elle ne vient pas de l'extérieur comme dans le drame symboliste, elle est tapie en euxmêmes. C'est de cette manière que le dramaturge renouvelle le concept de la tragédie, qui, le cas échéant, loin dêtre spectaculaire, n’en est pas moins émouvante.

La première pièce de Pellerin, jouée tout d'abord au Théâtre des Mathurins en 1922 (sur l'affiche figure également la fameuse Martine de Bernard) et puis, un an plus tard, à la Baraque de la Chimère, a joui d'un succès retentissant (elle a dépassé une centaine de représentations), tant s'en faut, car elle annonçait une nou- 
velle dramaturgie tournant le dos à la vieille charpente du théâtre mélodramatique. Pour la majorité écrasante, la critique réserve à la réalisation scénique du drame des mots pleins d'enthousiasme. Plusieurs rapporteurs, tels Lucien Besnard ou Jeanne Catulle Mendès, ne manquent pas non plus de faire ressortir les éléments novateurs du texte même : l'originalité de la plume de l'auteur de Maya résiderait avant tout dans la soustraction de celui-ci au carcan du rationalisme au profit de la vivisection des méandres ombrageux de l'âme humaine, le drame ne se fondant plus "sur l'événement mais sur l'état d'âme des protagonistes » (Lioure, 1973 : 133). Il n'est donc pas étonnant que les relents d'onirisme empiètent constamment sur le réel dans le drame, car l'inconscient ne peut se déployer librement que dans une atmosphère étrange et inexplicable. La raison et la langue ne peuvent y avoir accès et le silence prend inévitablement le relais. La montée de la pièce va du réalisme à l'irréalité imaginaire, les deux s'imbriquant au cours de l'action. C'est ce va-et-vient entre ces deux mondes apparemment distincts et surtout la matérialisation du subconscient des personnages qui fait du texte de Pellerin une œuvre indiscutablement originale à l'époque : «ce n'est pas la première fois bien sûr que la matière des songes est portée sur la scène, mais il y a peu d'exemples de pièces jouant continûment du seul ressort de la matérialisation de figures évoquée par le discours intérieur » (Lieber, 1987 : 369).

L’écrivain français semble réaliser dans son œuvre l'esthétique « intimiste " prônée par Baty, c'est pour cette raison qu'il a recours aux silences qui sont plus éloquents que les paroles des personnages tragiques (Baty, 1926: 145-183). Ces derniers vivent dans le royaume de Sire le Mot, où tout est dit ; en conséquence, ils sont privés de mystère, élément essentiel à la subsistance humaine, recherché par le fondateur $\mathrm{du}$ « Cartel » théâtral. En revanche, les silences évoquent l'existence d'une autre vie, peut-être plus vraie, comme le confirmeraient les surréalistes, car libérée du joug réducteur et déformant du logos. Pellerin cherche alors, sous les mots débités par ses protagonistes, les non-dits, les angoisses refoulées, la solitude, enfin l'ennui thétique (Cf. Rykner, 1996 : 256). Comme le remarque à juste titre Henri Bidou « dans les silences, leur pensée vagabonde, et le piquant de la pièce, c'est que cette pensée se matérialise et nous apparaît. Nous entendons les paroles non prononcées, nous voyons, pareils à des fantômes, les interlocuteurs imaginaires » (1922:101).

Les silences ne sont donc pas, comme le dit malicieusement Pierre-Aimé Touchard, "dignes de la réflexion silencieuse des spectateurs " (1938: 170), car en explorant le mental de ses personnages, Pellerin désire ouvertement faire parler leur inconscient à la manière de Jean-Jacques Bernard :

Un grand nombre d'hommes et de femmes, me semble-t-il, vivent des vies distinctes; l'une qui parle, gesticule, rit, pleure, crie, fait beaucoup de bruit, tient beaucoup de place; l'autre (la vraie) peut-être qui pense et rêve en sourdine, tout au fond de nous-mêmes, qui ne veut ou ne peut s'exprimer au grand jour qui n'ose, qui se cache - qui est notre silence (Cf. Kaczmarek, 2003 : 90). 
L'auteur lui-même ne tarde pas à expliquer les motifs qui l'ont poussé à écrire sa pièce : « j'ai simplement essayé de montrer tout ce qui se passe derrière la façade en paroles d'une existence quelconque... » (1922: 70). En le faisant, Pellerin conçoit une nouvelle approche de personnage, loin de la tradition "cartésienne ", qui scrute "le sentiment vague de notre corps" (Vogelweith, 1971 : 112) au détriment des sacro-saintes règles de la psychologie. Cette descente dans les profondeurs de l'âme humaine correspond bien aux recherches de Baty qui rejette vigoureusement l'idée selon laquelle tout homme reste analysable, celui-ci s'esquivant constamment à être cerné par la raison d'ailleurs depuis quelque temps discréditée :

L'homme en vérité dépasse de toutes parts ce schéma de l'homme. Sa vie consciente est baignée de vie inconsciente ou consciente seulement à demi. Il n'est pas seulement l'idée claire qu'il a de lui-même, mais ses rêves obscurs, sa mémoire endormie, ses instincts refoulés; dans l'ombre de son âme habitent ses ancêtres, l'enfant qu'il a été, les autres hommes qu'il aurait pu être. Tout cela n'affleure quà peine, par éclairs, dans le champ de sa conscience; cette vie obscure conditionne cependant son autre vie (Baty, 1945 : 107).

\section{BIBLIOGRAPHIE}

Baty G. 1926. Le masque et l'encensoir. Introduction à une esthétique du théâtre. Paris. Librairie Bloud \& Gay.

Baty G. 1945. Témoignages, études. Paris. Éditions du Pavois.

Bernard J.-J. 1931. De la valeur du silence dans les arts du spectacle. Masques. $25^{\mathrm{e}}$ cahier. 2330.

Bernard J.-J. 1958. Mon ami le Théâtre. Paris. Albin Michel.

Bidou H. 1922. La revue de presse. Bulletin de la Chimère. 99-103.

Jenkenne V. Note in : Le Journal de Liège. Bulletin de la Chimère. 114-115.

Kaczmarek T. 2003. Les hésitations théâtrales de Jean-Victor Pellerin. In : Queffélec Ch., Colin R. P. Écrire la rupture. Lyon. Éditions du Lérot. 81-101.

Lieber G. 1987. Gaston Baty et ses auteurs : le théâtre d'évasion. Thèse du doctorat d'État ès Lettres et Sciences Humaines. Université Paris X-Nanterre.

Lioure M. 1973. Le Drame de Diderot à Ionesco. Paris. Armand Colin.

Maeterlinck M. 1986. Le Trésor des humbles. Bruxelles. Éditions Labor.

Pellerin J.-V. 1922. À propos d'Intimité. Bulletin de la Chimère. 69-70.

Pellerin J.-V. 1924. Intimité. In : Masques. $17^{\mathrm{e}}$ cahier.

Raymond M. 1943. Le Jeu retrouvé. Montréal. Éditions de l'Arbre.

Rykner A. 1996. L'envers du théâtre. Dramaturgie du silence de l'âge classique à Maeterlinck. Paris. Librairie José Corti.

Rykner A. 2001. Le théâtre du silence. In : Corvin M. Dictionnaire encyclopédique du théâtre. Paris. Larousse.

Rousseaux Ph. 2003. Fonction du silence en pédagogie : une dimension performative. Éduquer.

En ligne, URL : http://rechercheseducations.revues.org/211, consulté le 26/07/2017.

Sarraute N. 1956. Lère du soupçon. Paris. Gallimard. 
Touchard P.-A. 1928. Dionysos. Apologie pour le Théâtre. Paris. Éditions Montaigne.

Vaïs M. 1978. L’écrivain scénique. Montréal. Presses de l’Université du Québec.

Vogelweith G. 1971. Le Personnage et ses métamorphoses dans le théâtre de Strindberg. Lille. Service de reproduction des thèses de l'Université de Lille III.

\section{Intimacy by Jean-Victor Pellerin, or in search of "theatre of the silence”}

ABSTRACT: Jean-Victor Pellerin was a representative author of the "school of silence" or, as some called it, „art of unexpressed”, in which emotions are implied in gestures, fragments of speach, and silence. In his first drama Intimacy (1922) the French playwrighter presents a bourgeois marriage living a monotonous life. The characters discuss trivial things of everyday life, they talk to each other, but there is no communication between them, because each of them is closed in their own world. Nobody is listening one another and words are spoken only in order to fill their existential emptiness. The writer is focused on the dialogue between the characters in the context of what they hide and what they do not want to tell themselves. People who appear to them are not flesh and blood human beings but they are the reflection of their own disturbed personality only. In this manner the writer focuses on the inner life of his solitary, their nostalgia for youth or even their hidden sexual motives. This is why the silence grows into the main element of the drama. Intimacy is one of the finest examples of „intimist aesthectic” developed by the avant-garde theater creator Gaston Baty who rejected the predominance of the word for discovering the mysterious world of the spiritual man.

Keywords: Jean-Victor Pellerin, Gaston Baty, theatre of the silence, soliloquy, loneliness. 